\title{
LOCALIZATIONS OF HNP RINGS
}

BY

\section{JAMES KUZMANOVICH}

ABSTRACT. In this paper it is shown that every hereditary Noetherian prime ring is the intersection of a hereditary Noetherian prime ring having no invertible ideals with a bounded hereditary Noetherian prime ring in which every nonzero twosided ideal contains an invertible two-sided ideal. Further, it is shown that this intersection corresponds to a decomposition of torsion modules over such a ring; if $R$ is an HNP ring with enough invertible ideals, then this decomposition coincides with that of Eisenbud and Robson.

If $M$ is a maximal invertible ideal of $R$ where $R$ is as above, then an overring of $R$ is constructed which is a localization of $R$ at $M$ in a "classical sense"'; that is, it is a ring of quotients with respect to a multiplicatively closed set of regular elements satisfying the Ore conditions. The localizations are shown to have nonzero radical and are also shown to satisfy a globalization theorem.

These localizations are generalizations of ones constructed by A. V. Jategaonkar for HNP rings with enough invertible ideals.

The main tool used throughout the paper is that of a ring of quotients with respect to an idempotent kernel functor. Each such kernel functor is determined by a filter of right ideals, or equivalently a class of cyclic modules. The technique used in construction of each of the above rings will be to define a class of cyclic modules by the presence or absence of certain composition factors, form the ring of quotients with respect to the kernel functor thus defined, and then show that the ring of quotients has the desired properties.

$\operatorname{Mod}\left(R_{R}\right)\left(\operatorname{Mod}\left({ }_{R} R\right)\right)$ will denote the category of right (left) $R$-modules, and all ring theoretic conditions (Noetherian, Artinian, hereditary, etc.) will be twosided unless otherwise stated. During much of the remainder of this paper $R$ will be a hereditary Noetherian prime ring (we will often shorten this to HNP ring). In this situation $R$ will be a prime Goldie ring and hence will have a simple Artinian classical quotient ring which we will call $Q$. We will assume throughout that $R$ is not Artinian; that is, $R \neq Q$.

The author would like to thank the Faculty Research Committee of the University of Northern Colorado for a grant in support of this research.

1. Preliminaries. A right $R$-submodule of $Q$ is called a right $R$-ideal (fractional) if $I$ contains a regular element of $Q$ and if there is a regular element $b$ of

Received by the editors June 4, 1971.

AMS (MOS) subject classifications (1970). Primary 16A08, 16A14.

Key words and phrases. Dedekind prime ring, hereditary Noetherian prime ring, ring of quotients, overring, hereditary torsion theory, idempotent kernel functor. 
$Q$ such that $b I \subset R$. The reader may want to refer to Jacobson [8] who has a full account of these definitions. If $I \subset R$, then $I$ is called integral. If $I$ is both a left and a right $R$-ideal, then $I$ is called an $R$-ideal. If $I$ is a right (left) $R$-ideal, define $I^{*}(* I)$ by $I^{*}=\{q \in Q: q I \subset R\} \quad(* I=\{q \in Q: I q \subset R\})$. Note that $I^{*}$ is isomorphic to $\operatorname{Hom}_{R}(I, R)$, the dual of $I$. If $I$ is an $R$-ideal, then it is not in general true that ${ }^{*} I=$ $I^{*}$. An $R$-ideal $I$ is called invertible if $\left(I^{*}\right) I=I\left({ }^{*} I\right)=R$. In this case it will be true that $I^{*}={ }^{*} I$, denote it by $I^{-1}$. An HNP ring in which every $R$-ideal is invertible is called a Dedekind prime ring (Robson [15]).

If $m$ is an element of a right $R$-module $M$, then $m$ is called a torsion element if $m b=0$ for a regular element $b$ of $R$. Levy [12] showed that if $R$ had a classical right quotient ring, then the set of all torsion elements of $M$, denoted by $t(M)$, was a submodule. Later in the paper we will be concerned with various other torsion submodules relative to other torsion theories, but when the word torsion is used without adjectives, we will mean the torsion just defined.

Webber [17] has shown that if $I$ is an essential right ideal of an HNP ring $R$, then $R / I$ is Artinian. Since in a prime Goldie ring a right ideal is essential if and only if it contains a regular element, it easily follows that a finitely generated torsion module over an HNP ring is Artinian. Hence a finitely generated torsion module would have a composition series; in particular if $I$ is an essential right ideal, then $R / I$ has a composition series.

Goldman [7] has called a covariant functor $\sigma$ from $\operatorname{Mod}\left(R_{R}\right)$ to $\operatorname{Mod}\left(R_{R}\right)$ an an idempotent kernel functor if $\sigma$ satisfies the following properties:

(1) $\sigma(M) \subset M$ for all right $R$-modules $M$.

(2) If $f \in \operatorname{Hom}_{R}\left(M^{\prime}, M\right)$ for right $R$-modules $M^{\prime}$ and $M$, then $f\left(\sigma\left(M^{\prime}\right)\right) \subset \sigma(M)$ and $\sigma(f)$ is $\left.f\right|_{\sigma\left(M^{\prime}\right)}$.

(3) If $M^{\prime} \subset M$, then $\sigma\left(M^{\prime}\right)=\sigma(M) \cap M^{\prime}$.

(4) $\sigma(M / \sigma(M))=0$ for every right $R$-module $M$.

Note that $\mathcal{G}_{\sigma}=\left\{M_{R}: \sigma(M)=M\right\}$ is a hereditary torsion class in the sense of Dickson [2]. In fact the concepts of torsion theories and kernel functors are equivalent in the sense that each torsion theory gives rise to a kernel functor whose torsion class is that of the original torsion theory. $\sigma(M)$ is called the $\sigma$-torsion submodule of $M$. If $\sigma(M)=M$, then $M$ is called $\sigma$-torsion. If $\sigma(M)=0$, then $M$ is called $\sigma$-torsionfree. See also Gabriel [6], Maranda [13], Fuchs [5], and Walker and Walker [16]. Lambek [11] has recently given a very good account of torsion theories (kernel functors) and rings of quotients. There is a filter, $\mathcal{T}_{\sigma}$, of right ideals associated with $\sigma$ defined by $\mathcal{T}_{\sigma}=\left\{I_{R} \subset R: R / I \in \mathcal{I}_{\sigma}\right\}$. Then $\mathcal{I}_{\sigma}$ determines $\sigma$ in the following sense: if $x \in M_{R}$, then $x \in \sigma(M)$ if and only if there exists $I \in \mathcal{J}_{\sigma}$ such that $x I=$ 0 . See Gabriel [6] and Goldman [7]. Given a right $R$-module $M$ such that $\sigma(M)=0$, Goldman defines the module of quotients of $M$ with respect to $\sigma, Q_{\sigma}(M)$, in the following way: let $E$ be an injective hull of $M$, then let $Q_{\sigma}(M)=\{x \in E:[x+M]$ is an 
element of $\sigma(E / M)\} . Q_{\sigma}(M)$ is unique up to a unique isomorphism over $M$. If $\sigma\left(R_{R}\right)=0$, then $Q_{\sigma}(R)$ has a unique ring structure extending the $R$-module structure. $Q_{\sigma}(R)$ (often shortened to $Q_{\sigma}$ ) is called the ring of quotients of $R$ with respect to $\sigma$. In our situation where $R$ is a prime Goldie ring with classical quotient ring $Q, Q_{R}$ is the injective hull of $R$ and the ring of quotients of $R$ with respect to the filter of all essential right ideals of $R$. If $\sigma$ is an idempotent kernel functor such that $\sigma(R)=0$, then by the above construction it is clear that $Q_{\sigma}=\{q \in Q: q I \subset R$ for some $\left.I \in \mathcal{T}_{\sigma}\right\}$, since $Q$ is the injective hull of $R$. It can be seen that $Q_{\sigma}$ is in fact a subring of $Q$. If every right ideal in $\mathfrak{T}_{\sigma}$ is essential in $R$, then $q \in Q_{\sigma}$ if and only if $q \in I^{*}$ for some $I \in \mathcal{T}_{\sigma}$. This is summarized in the following proposition.

Proposition 1.1. Let $\sigma$ be an idempotent kernel functor such that $\sigma(R)=0$ and every right ideal in $\mathfrak{T}_{\sigma}$ is essential in $R$. Then $Q_{\sigma}=\bigcup I^{*}$ where $I$ ranges over the elements of $\mathcal{T}_{\sigma}$.

An idempotent kernel functor $\sigma$ is said to have property $(T)$ if it satisfies any of the equivalent conditions of the following theorem; the theorem is basically Theorem 4.3 of [7].

Theorem 1.2. Let $\sigma$ be an idempotent kernel functor. The following statements are equivalent:

(1) Every $Q_{\sigma}$-module is $\sigma$-torsion free.

(2) $I Q_{\sigma}=Q_{\sigma}$ for every $I \in \mathcal{J}_{\sigma}$.

(3) $M \otimes_{R} Q_{\sigma} \simeq Q_{\sigma}(M)$ for every right $R$-module $M$.

In this paper we will be concerned exclusively with HNP rings and with kernel functors $\sigma$ such that $Q_{\sigma}$ is an overring of $R$. It is not hard to see that over a hereditary Noetherian ring every kernel functor has property $(T)$. In fact in our situation every $Q_{\sigma}$ is an HNP ring and is flat as an $R$-module (both left and right); see [10]. Let $I$ be an integral right $R$-ideal and suppose that $I Q_{\sigma}=Q_{\sigma}$. Take $r \in R$. Then it is easy to see that there is a $J$ in $\mathcal{J}_{\sigma}$ such that $r J \subset I$. Hence $\sigma(R / I)=R / I$ and $I \in \mathcal{J}_{\sigma}$. Together with (2) of Theorem 1.2 we have that $I \in \mathcal{T}_{\sigma}$ if and only if $I Q_{\sigma}=Q_{\sigma}$. If $I Q_{\sigma}$ $=Q_{\sigma}$, then we will say that $I$ explodes in $Q_{\sigma}$ and in general $I Q_{\sigma}$ is called the expansion of $I$ in $Q_{\sigma}$. If $J$ is an integral right $Q_{\sigma}$-ideal, then $J^{c}=J \cap R$ is called the contraction of $J$ to $R$. Proposition 4.6 of [7] shows that if $\sigma$ has property $(T)$, then every right ideal of $Q_{\sigma}$ is the expansion of its contraction. This property will be important in the rest of the paper.

2. $R$ as an intersection, decompositions of torsion modules. In this section it is shown that $R$ is the intersection of two overrings, one of which has no invertible ideals while the other is bounded with enough invertible ideals. These rings are constructed as rings of quotients with respect to two torsion theories. It is also shown that each torsion module over $R$ is the direct sum of the two torsion submodules corresponding to the above overrings. This decomposition generalizes the 
fact that over a Dedekind prime ring a finitely generated torsion module is a direct sum of a completely faithful module and a bounded module, as a result of Eisenbud and Robson [4].

If $M$ is a right $R$-module, define $b(M)$, the invertible torsion submodule, by $b(M)=\{m \in M: m B=0$ for some invertible two-sided ideal of $R, B\}$. If $f: M^{\prime} \rightarrow M$ is an $R$-homomorphism of right $R$-modules, let $b(f)$ be the restriction of $f$ to $b\left(M^{\prime}\right)$.

Proposition 2.1. $b$ is an idempotent kernel functor.

Proof. Let $M$ be a right $R$-module. It will first be shown that $b(M)$ is a submodule. Let $x$ and $y$ be elements of $b(M)$. Then there are invertible two-sided ideals of $R, A$ and $B$, such that $x A=0$ and $x B=0 . A B$ is an invertible ideal contained in $A \cap B$ so that $(x+y) A B \subset(x+y)(A \cap B)=0$ and $x+y \in b(M)$. If $r \epsilon$ $R$, then $(x r) A=x(r A) \subset x A=0$ and $x r \in b(M)$. Hence $b(M)$ is a submodule of M. Let $f: M^{\prime}$ $\rightarrow M$ be an $R$-homomorphism of right $R$-modules. Take $x \in b\left(M^{\prime}\right)$; then there is an invertible two-sided ideal $B$ of $R$ such that $x B=0 . f(x) B=f(x B)=0$ so that $f(x) \epsilon$ $b(M)$. Hence $f\left(b\left(M^{\prime}\right)\right) \subset b(M)$. It is clear that if $M^{\prime} \subset M$, then $b\left(M^{\prime}\right)=M^{\prime} \cap b(M)$. Thus $b$ is a kernel functor. Let $M$ be a right $R$-module and consider $M / b(M)$. Suppose that $[x+b(M)] \in M / b(M)$ is $b$-torsion. Then there is a two-sided invertible ideal $B$ of $R$ such that $x B \subset b(M) . R$ is Noetherian so that $B=r_{1} R+\cdots+r_{n} R$ and $x B=x r_{1} R+\cdots+x r_{n} R$. Let $A_{i}(i=1, \cdots, n)$ be a two-sided invertible ideal of $R$ such that $x r_{i} A_{i}=0$. Then $x\left(B A_{1} \cdots A_{n}\right)=\left(x r_{1} R+\cdots+x r_{n} R\right)\left(A_{1} \cdots A_{n}\right)=$ $x r_{1} A_{1} \cdots A_{n}+\cdots+x r_{n} A_{1} \cdots A_{n} \subset x r_{1} A_{1}+\cdots+x r_{n} A_{n}=0$. Therefore, since $B A_{1}$ $\cdots A_{n}$ is invertible, $x \in b(M)$ and $[x+b(M)]=[0+b(M)]$ and $M / b(M)$ is $b$-torsion free. That is, $b$ is idempotent.

Lemma 2.2. $\mathcal{T}_{b}=\{I: I$ is a large right ideal containing an invertible two-sided ideal of $R$ \}.

Proof. $\mathcal{T}_{b}=\left\{I_{R} \subset R: b(R / I)=R / I\right\}$. Hence if $I \in \mathfrak{T}_{b}$, then there is an invertible two-sided ideal of $B$ such that $1 \cdot B \subset I$. $I$ is large since all two-sided ideals are large.

Form the quotient ring with respect to $b, Q_{b}$. By Proposition $1.1 Q_{b}$ is just the union of all $I^{*}$ where $I$ is an element of $\mathcal{T}_{b}$. In fact, more can be said as is seen in the following proposition.

Proposition 2.3. $Q_{b}$ is the union of all $B^{-1}$ where $B$ is an invertible twosided ideal of $R$. Furthermore, the quotient ring resulting from considering the invertible torsion theory in the category of left $R$-modules is precisely $Q_{b}$.

Proof. $Q_{b}=\bigcup\left\{I^{*}: I \in \mathfrak{T}_{b}\right\}$. If $I \in \mathfrak{T}_{b}$, then by Lemma $2.2 I \supset B$, where $B$ is an invertible two-sided ideal of $R$. But $B \subset I$ implies that $I^{*} \subset B^{*}$ and thus it follows that $Q_{b}=\bigcup\left\{B^{*}: B\right.$ a two-sided invertible ideal of $\left.R\right\}$.

The furthermore follows since the quotient ring with respect to the analogous left torsion theory will just be the union of all ${ }^{*} B$ where $B$ is an invertible ideal of 
$R$. But if $B$ is invertible, then ${ }^{*} B=B^{-1}=B^{*}$ so that taking unions yields equality.

Call a right (left) $R$-module $M$ completely $b$-torsion free $(c b f)$ if every submodule of every factor module of $M$ is $b$-torsion free. A right (left) integral $R$-ideal $I$ is called $c b f$ if $R / I$ is $c b f$. Note that $I$ will be $c b f$ if and only if $R / I$ has no $b$ torsion composition factors.

By the results of the first section we know that every right (left) integral $Q_{b}$ ideal is the expansion of its contraction and that a right (left) $R$-ideal explodes in $Q_{b}$ if and only if it contains an invertible two-sided ideal of $R$. (The parenthetical version follows from Proposition 2.3.) The following proposition expands on the relationships between the ideals of $R$ and those of $Q_{b}$.

Proposition 2.4. $\left(I Q_{b}\right)^{c}$

(i) If $I$ is a cbf right (left) integral $R$-ideal, then $R / I \simeq Q_{b} / I Q_{b}$ and $I=$

(ii) Let I be an integral right (left) $Q_{b}$-ideal. Then $I^{c}$ is cbf and $R / I^{c} \simeq$ $Q_{b} / I$.

(iii) If $I$ is a cbf integral right (left) $R$-ideal, then $I=\left(I Q_{b}\right)^{c}$.

(iv) If $M$ is a cbf integral $R$-ideal, then $Q_{b} M=M Q_{b}=Q_{b} M Q_{b}$ is a proper $Q_{b}$ ideal and $R / M \simeq Q_{b} / M Q_{b}$ (as rings).

Proof. (i) Let $I$ be an integral right $R$-ideal such that $R / I$ is $c b /$. Then $R / I$ has no $b$-torsion composition factors and hence $I+B=R$ for every invertible twosided ideal $B$. Thus $I B^{-1}+B B^{-1}=R B^{-1}$ or $I B^{-1}+R=B^{-1}$. Since $Q_{b}$ is the union of all such $B^{-1}$, it follows that $I Q_{b}+R=Q_{b}$. The second isomorphism theorem yields that $Q_{b} / I Q_{b}$ is isomorphic to $R /\left(I Q_{b}\right)^{c}$. Let $x \in\left(I Q_{b}\right)^{c}$; then $x \in$ $I B^{-1}$ for some invertible two-sided integral $R$-ideal $B$ and hence $x B \subset I$. That is, $\left(I Q_{b}\right)^{c} / I$ is $b$-torsion; this implies that $I=\left(I Q_{b}\right)^{c}$ since $R / I$ had no $b$-torsion composition factors. Combining results yields that $R / I \simeq Q_{b} / I Q_{b}$.

(ii) The proof of (ii) will be an induction argument on the composition length of $R / I^{c}$ where $I$ is an integral right $Q_{b}$-ideal. If the composition length of $R / I^{c}$ is one, then $R / I^{c}$ is simple. $R / I^{c}$ must be $b$-torsion free (and hence $c b f$ ) for otherwise $I^{c}$ would explode in $Q_{b}$. Hence by (i) $R / I^{c} \simeq Q_{b} / I^{c} Q_{b}=Q_{b} / I$. Now suppose that the result is true whenever the composition length of the contraction is $k-1$ and assume that $I$ is such that the composition length of $R / I^{c}$ is $k$. Then there is a composition series for $R / I^{c}$ of the form $I^{c}=J_{0} \subset \cdots \subset J_{k}=R$. By the second isomorphism theorem $(I+R) / I \simeq R / I^{C}$ and $(I+R) / I$ is an $R$-submodule of the $Q_{b}$-module $Q_{b} / I . Q_{b}$-modules are $b$-torsion free so that $R / I^{c}$ is $b$-torsion free; hence $J_{1} / I^{C}$ is also $b$-torsion free. $\left(J_{1}+I\right) / I \simeq J_{1} / I^{c}$ is thus a $b$-torsion free $R$ submodule of $Q_{b} / I$ and hence is a $Q_{b}$-submodule of $Q_{b} / I . I+J_{1}$ is then a right $Q_{b}$-ideal whose contraction $\left(J_{1}\right)$ has composition length $k-1$. By the induction hypothesis it follows that $R / J_{1}$ is $c b f$. Hence $R / I^{c}$ has no $b$-torsion composition 
factors. By (i) $R / I^{c} \simeq Q_{b} / I Q_{b}=Q / I$ and (ii) is proven.

(iii) Let $I$ be a $c b f$ integral right $R$-ideal. Certainly $I \subset\left(I Q_{b}\right)^{c}$. By (i) the composition length of $R / I$ is equal to the composition length of $Q_{b} / I Q_{b}$ as a right $R$-module; but by (ii) this is the same as the composition length of $R /\left(I Q_{b}\right)^{c}$. Hence $I=\left(I Q_{b}\right)^{c}$.

Note that the parenthetical versions of (i), (ii), and (iii) follow by Proposition 2.3 .

(iv) Let $M$ be a $c b f$ integral $R$-ideal and suppose that $Q_{b} M Q_{b}=Q_{b}$. Then $\sum x_{i} m_{i} y_{i}=1$ for $x_{i}$ 's and $y_{i}$ 's in $Q_{b}$ and $m_{i}$ 's in $M$. By an argument similar to that of Proposition 2.1 there is an invertible integral $R$-ideal $B$ such that all the $x_{i}$ 's and $y_{i}$ 's are in $B^{-1} \cdot B^{2}=B \cdot 1 \cdot B=B\left(\sum x_{i} m_{i} y_{i}\right) B=\sum B x_{i} m_{i} y_{i} B . B x_{i}$ and $y_{i} B$ are contained in $R$ for all $i$ so that $B^{2}=\sum B x_{i} m_{i} y_{i} B$ is contained in $M$. This contradicts the fact that $R / M$ is cbf since $B^{2}$ is invertible. Hence $Q_{b} M Q_{b} \neq$ $Q_{b}$. Let $N=\left(Q_{b} M Q_{b}\right)^{c}$. Every integral $Q_{b}$-ideal is the expansion of its contraction so that $N Q_{b}=Q_{b} M Q_{b}=Q_{b} N$. $N$ is finitely generated as a right $R$-module so that $N Q_{b}=\left(\Sigma x_{i} R\right) Q_{b}=\Sigma x_{i} Q_{b}$ where the $x_{i}$ 's are in $N . Q_{b}\left(M Q_{b}\right)=N Q_{b}$ so that for each $i$ there is an invertible two-sided ideal $B_{i}$ of $R$ such that $x_{i} \in B_{i}^{-1}\left(M Q_{b}\right)$. Again it is easy to see that there is a single invertible ideal $B$ such that $x_{i} \epsilon$ $B^{-1}\left(M Q_{b}\right)$ for all $i$. As a result $M Q_{b} \supset B\left(\Sigma x_{i} Q_{b}\right)=B N Q_{b}=B Q_{b} N=Q_{b} N=N Q_{b}$. Thus $M Q_{b}=N Q_{b}$. Similarly $Q_{b} M=Q_{b} N=Q_{b} M Q_{b}=M Q_{b}$. By (iii) it follows that $M=N$. The map $f: R / M \rightarrow Q_{b} / M Q_{b}$ defined by $f([r+M])=\left[r+M Q_{b}\right]$ is a welldefined and a one-to-one ring homomorphism since $\left(M Q_{b}\right)^{c}=M$. It is onto since $R+$ $M Q_{b}=Q_{b}$ as in (i).

Corollary 2.5. Every cyclic $Q_{b}$-module is cbf as an R-module.

Theorem 2.6. $Q_{b}$ is a hereditary Noetherian prime ring with no invertible ideals. The one-sided (two-sided) $Q_{b}$-ideal lattices are isomorphic to the onesided (two-sided) lattices (lattice) of cbf R-ideals, and the corresponding factor modules (rings) are isomorpbic.

Proof. Every overring of $R$ is hereditary and Noetherian (Proposition 1.6 of [10]); hence so is $Q_{b}$. The last statement is a restatement of parts of Proposition 2.4. It remains to be shown that $Q_{b}$ has no invertible ideals.

Let $I$ be an invertible (two-sided) ideal of $Q_{b}$. Then $I^{-1}=\left\{q \in Q: q I \subset Q_{b}\right\}$. We will first show that $I^{-1}=Q_{b}\left(I^{c}\right)^{*} . I=I^{c} Q_{b}$ so that $Q_{b}\left(\left(I^{c}\right)^{*}\right) I^{c} Q_{b}$ is contained in $Q_{b} R Q_{b}=Q_{b}$ and $Q_{b}\left(I^{c}\right)^{*} \subset I^{-1}$. Let $q$ be in $I^{-1}$. Then $q I \subset Q_{b}$ and in particular $q I^{C} \subset Q_{b} \cdot q I^{C}$ is a finitely generated $R$-submodule of $\cdot Q_{b}$ so that by a simple argument similar to that in Proposition 2.1 there is an invertible integral $R$-ideal $B$ such that $q I^{c} \subset B^{-1}$. Hence $q I^{c}\left(I^{c}\right)^{*} \subset B^{-1}\left(I^{c}\right)^{*} .1 \in\left(I^{c}\right)\left(I^{c}\right)^{*}$ so that $q \epsilon$ $B^{-1}\left(I^{c}\right)^{*}$; that is, $q \in \mathrm{Q}_{b}\left(I^{c}\right)^{*}$. As a result both containments hold and we have that $I^{-1}=Q_{b}\left(I^{c}\right)^{*}$. 
$I^{c}$ is $c b f$ so that it cannot be contained in any invertible ideal of $R$. Then there is an integer $k$ such that $\left(I^{c}\right)^{k}$ is idempotent by Proposition 4.5 of Eisenbud and Robson [3]. By (iv) of Proposition 2.4I= $I^{c} Q_{b}$ so from (iii) of Proposition 2.4 it follows that $\left(I^{k}\right)^{c}=\left(I^{c}\right)^{k}$ since $I^{k}=\left(I^{c}\right)^{k} Q_{b}$. The proof above shows that $Q_{b}\left(\left(I^{c}\right)^{k}\right)^{*}=\left(I^{k}\right)^{-1} .\left(I^{c}\right)^{k}$ is idempotent so that $\left(\left(\left(I^{c}\right)^{k}\right)^{*}\right)\left(I^{c}\right)^{k}$ by Lemma 1.5 of [3]. Hence $Q_{b}=\left(I^{k}\right)^{-1}\left(I^{k}\right)=Q_{b}\left(\left(\left(I^{c}\right)^{k}\right)^{*}\right)\left(I^{c}\right)^{k} Q_{b}=Q_{b}\left(I^{c}\right)^{k} Q_{b}=I^{k}$. Thus $I$ was not a proper ideal and $Q_{b}$ has no invertible ideals.

Lemma 2.7. Let $M$ be a right $Q_{b}$-torsion module. Then the structure of $M$ as an $R$-module is identical to the structure of $M$ as a $Q_{b}$-module; that is, every $R$ submodule of $M$ is a $Q_{b}$-submodule of $M$ and vice versa.

Proof. The fact that the result is true for cyclic $M$ is basically the content of (ii) of Proposition 2.4. In fact $x Q_{b}=x R$ for all $x$ in $M$ by the same reason. Since any $R$-submodule of $M$ is a sum of cyclic $R$-submodules of $M$, it follows that every $R$-submodule of $M$ is a sum of cyclic $Q_{b}$-submodules of $M$, and thus is a $Q_{b}$-submodule of $M$. Clearly, every $Q_{b}$-submodule is an $R$-submodule.

Proposition 2.8. Let $M$ be a simple right cbf $R$-module. Then the R-injective bull of $M$ is $c b f$.

Proof. $M$ is simple so that $M$ is isomorphic to $R / I$ for a maximal right ideal I. We have that $M=R / I \simeq Q_{b} / I Q_{b}$. The $Q_{b}$-injective hull $H$ of $Q_{b} / I Q_{b}$ is $Q_{b}$ torsion (in the sense of Levy) and is thus $Q_{b}$-divisible. Since $R$ is contained in $Q_{b}, H$ is $R$-divisible. By a theorem of Levy [12] $H$ is thus injective as an $R$-module. Thus $E$, the $R$-injective hull of $M$, is an $R$-submodule of $E$; by Lemma $2.7 E$ is in fact a $Q_{b}$-submodule of $H$. Let $N$ be an $R$-submodule of $E$, by Lemma $2.7 N$ is a $Q_{b}$-submodule of $E$ and hence $E / N$ is a $Q_{b}$-module. It follows that every $R$ submodule of $E / N$ is $b$-torsionfree and hence, since $N$ was arbitrary, that $E$ is $c b f$.

The following corollary is one of the main results of this section and will be used again in the paper.

Corollary 2.9. Let $B$ and $C$ be simple right $R$-modules such that $B$ is b-torsion and $C$ is cbf. Then $\operatorname{Ext}_{R}^{1}(B, C)=0$.

Proof. Ext ${ }^{1}(B, C)=0$ if and only if every short exact sequence of $R$-modules

$$
0 \rightarrow C \rightarrow A \rightarrow B \rightarrow 0
$$

splits. Assume that there is a sequence $(*)$ that does not split. $C$ is then an essential submodule of $A$ so that $E(C)=E(A)$ (injective hulls). By Proposition 2.8 $E(A)$ is a $c b f R$-module. But this leads to a contradiction since $B$ is a $b$-torsion submodule of $E(A) / C$.

Much of the remainder of this section is concerned with the construction of the 
ring of quotients which is complementary to the ring $Q_{b}$, the ring of quotients with respect to the torsion theory determined by the $c b f$ integral right $R$-ideals.

Let $\mathcal{T}_{c}=\left\{I_{R} \subset R: R / I\right.$ is $\left.c b f\right\}$. In review, if $M$ is a right $R$-module, then $c(M)=$ $\left\{x \in M: x I=0\right.$ for some $\left.I \in \mathcal{T}_{c}\right\}$.

Lemma 2.10. $\mathcal{T}_{c}$ determines a kernel functor.

Proof. Let $I \subset J \subset R$ be right ideals of $R$ with $I \in \mathcal{T}_{c}$. Then $R / I$ is $c b f$ and has no $b$-torsion composition factors; hence neither does $R / J$ since it is a homomorphic image of $R / I$; hence $J \in \mathcal{T}_{c}$. Now let $I$ and $J$ be elements of $\mathcal{T}_{c} . I /(I \cap J)$ $\simeq(I+J) / J$ which is contained in $R / J$ which has no $b$-torsion composition factors. $R / I$ has no $b$-torsion composition factors either; consequently neither does $R /(I \cap J)$ and $I \cap J \in \mathfrak{T}_{c}$. Take $I \in \mathcal{T}_{c}$ and $x \in R$. Let $(I: x)=\{r \in R: x r \in I\}$. It is easy to see that $R /(I: x) \simeq(I+x R) / I$ (take $[r+(I: x)]$ to $[x r+I]) . R / I$ has no $b$ torsion composition factors so that neither can $R /(I: x)$. Thus $(I: x) \in \mathcal{T}_{c}$ and there is an element of $\mathcal{T}_{c}$ which annihilates $[x+I]$ in $R / I$. We have shown that the three properties of Proposition 2.1 of [7] hold. It follows that $\mathcal{T}_{c}$ defines a kernel functor.

Lemma 2.11. Let $M$ be an Artinian right $c$-torsion module, then $M$ is cbf.

Proof. Let $x \in M$; then $x$ is $c$-torsion; that is, there exists $I \in \mathfrak{T}_{c}$ such that $x I=0$. Hence $x R$ which is a homomorphic image of $R / I$ is $c b f$. Let $x_{1}, \cdots, x_{n}$ be a set of generators for $M$; then $M=x_{1} R+\cdots+x_{n} R . M^{\prime}=x_{1} R \oplus \cdots \oplus x_{n} R$ is $c b f$ and hence $M$ which is a homomorphic image of $M^{\prime}$ is cbf.

The next proposition shows that $\mathcal{T}_{c}$ defines an idempotent kernel functor.

Proposition 2.12. $c$ is an idempotent kernel functor.

Proof. $c$ is a kernel functor by Lemma 2.10. By Theorem 2.5 of [7] $c$ will be idempotent if it can be shown that if $I \subset J \subset R$ are right ideals of $R$ such that $J \epsilon$ $\mathcal{T}_{c}$ and $J / I$ is $c$-torsion, then $I \in \mathcal{T}_{c}$. Let $I$ and $J$ be such. $J / I$ is Artinian and a cbf module by Lemma 2.11 so that $J / I$ has no $b$-torsion composition factors. $J \in \mathfrak{T}_{c}$ so that $R / J$ has no $b$-torsion composition factors. Hence $R / I$ has no $b$-torsion composition factors and $I \in \mathcal{T}_{c}$.

$c$ is an idempotent kernel functor so that we can construct the corresponding ring of quotients $Q_{c}$. By Proposition $1.1 Q_{c}$ is just the union of all $I^{*}$ where $I \epsilon$ $\mathcal{J}_{c}$. Before giving any properties of $Q_{c}$ it will be necessary to introduce a duality for finitely generated torsion modules which is due to G. M. Bergman [1] and P. M. Cohn. The following theorem is a restatement of Proposition 51 of Bergman [1] for the case of a hereditary Noetherian ring. The proof may be found there.

Theorem 2.13. Let $R$ be a bereditary Noetherian ring. There exists an antiisomorphism, a, between the categories of finitely generated right torsion $R$-modules and finitely generated left torsion $R$-modules, such that if $M$ is the cokernel of 
$P \stackrel{i}{\rightarrow} Q$ where $P$ and $Q$ are finitely generated projectives, then the anti-isomorphic image of $M, \alpha(M)$, will be isomorphic to the cokernel of the map $Q^{*} \stackrel{i^{*}}{\rightarrow} P^{*}$.

We will be concerned with the situation when $P$ and $Q$ are fractional $R$-ideals. Namely, if $I$ is an integral right $R$-ideal, then $a(R / I)$ is $I^{*} / R$ since $R^{*}=R$. In the following we will implicitly use that $I m \rightarrow * / R$ is an anti-isomorphism. We are of course also using the fact that $I^{*}$ is isomorphic to $I^{*}=\operatorname{Hom}_{R}(I, R)$. Note also that $I^{* *}=I$.

Lemma 2.14. Let $I$ be an integral left $R$-ideal such thai $R / I$ is cbf. Then $* I / R$ is cbf.

Proof. The proof is by induction on the composition length of $R / I$. Suppose $R / I$ is simple and $c b f$ but ${ }^{*} I / R$ is not $c b f .{ }^{*} I / R$ is simple so that this means that ${ }^{*} I / R$ is $b$-torsion. Then there is an invertible two-sided ideal $B$ of $R$ such that $\left({ }^{*} I / R\right) B=0$; that is ${ }^{*} I B \subset R$ or ${ }^{*} I B B^{-1}={ }^{*} I R={ }^{*} I \subset R B^{-1}=B^{-1}={ }^{*} B$. Taking duals yields that $I={ }^{* *} I \supset{ }^{* *} B=B$ contradicting the fact that $R / I$ was $c b f$. Hence $* I / R$ is $c b f$. Inductively suppose that the result holds for all $c b f$ left ideals $J$ such that the composition length of $R / J$ is less than or equal to $k$ and let $I$ be such that $R / I$ is $c b f$ and has composition length $k+1$. Then there is a left ideal $J$ such that $I \subset J \subset R$ where $R / J$ is $c b f$ and has composition length $k . R \subset{ }^{*} J \subset{ }^{*} I$. By the induction hypothesis it follows that ${ }^{*} J / R$ is $c b f$. Also note that ${ }^{*} I /{ }^{*} J$ is just $a(J / I)$ which is simple. Again by the induction hypothesis ${ }^{*} I /{ }^{*} J$ is $c b f$. As a result $* I / R$ has no $b$-torsion composition factors and is $c b f$. The result follows by induction.

Proposition 2.15. $Q_{c}$ is the ring of quotients of $R$ with respect to both the left and right $c$-torsion theories.

Proof. To prevent confusion let ${ }_{c} \mathcal{T}$ be the family of left ideals that generates the $c$-torsion theory in $\operatorname{Mod}\left({ }_{R} R\right)$ and let ${ }_{c} Q$ be the corresponding ring of quotients. Let $I \in{ }_{c} \mathfrak{T}$; by Lemma $2.14{ }^{*} I / R$ is $c b f$ and hence is $c$-torsion. Consequently if $x \in I^{*}$, then there is a $J \in \mathfrak{T}_{c}$ such that $x J \subset R$. But this just says that $x \in J^{*} . Q_{c}$ is just the union of all such $J^{*}$ so that $x \in Q_{c}$; however, ${ }_{c} Q$ is just the union of all such ${ }^{*} I$. Hence ${ }_{c} Q \subset Q_{c}$; similarly it follows that $Q_{c} \subset{ }_{c} Q$ and $Q_{c}={ }_{c} Q$.

Lemma 2.16. Let $M \subset N$ be $R$-submodules of a $Q_{c}$-module $K$. If $M Q_{c}=N Q_{c}$, then $N / M$ is c-torsion.

Proof. Take $x \in N, x$ is then in $N Q_{c}=M Q_{c}$ so that $x=\Sigma m_{i} y_{i}$ where the $m_{i}$ 's are in $M$ and the $y_{i}{ }^{\prime}$ s are in $Q_{c} . Q_{c}$ is the union of all elements of $\mathfrak{T}_{c}$; therefore, for each $i$ there is an $I_{i} \in \mathfrak{T}_{c}$ such that $y_{i} \in I_{i}^{*}$. Hence $y_{i} \in I_{1}^{*}+\cdots+I_{n}^{*}$ $=\left(I_{1} \cap \ldots \cap I_{n}\right)^{*}$ for each $i . I=I_{1} \cap \ldots \cap I_{n} \in \mathcal{T}_{c} . x I=\left(\Sigma m_{i} y_{i}\right) I=\Sigma m_{i} y_{i} I \subset$ $\sum m_{i} R \subset M$; therefore, $[x+M]$ was $c$-torsion in $N / M$. $x$ was arbitrary in $N$ so that $N / M$ is $C$-torsion. 
Proposition 2.17. (i) Let $I \in \mathfrak{T}_{b}$, that is, let $R / I$ be b-torsion. Then $R / I \simeq$ $Q_{c} / I Q_{c}$ and $I=\left(I Q_{c}\right)^{c}$.

(ii) Let $B$ be a two-sided of $\mathcal{T}_{b}$. Then $Q_{c} M Q_{c} \neq Q_{c}$ and $Q_{c} M=Q_{c} M Q_{c}=$ $M Q_{c}$. Furthermore, $R / M$, and $Q_{c} / M Q_{c}$ are isomorphic as rings.

Proof. (i) Let $I \in \mathcal{T}_{b}$; $I$ will then contain an invertible two-sided ideal $B$ of $R$. If $J \in{ }_{c} \mathcal{T}$ ( $J$ is a $c b f$ integral left $R$-ideal), then $R=J+B \subset J+I$. It follows that ${ }^{*} J \subset R^{*} J=J^{*} J+I^{*} J \subset R+I Q_{c} \cdot Q_{c}$ is the union of all such ${ }^{*} J$ so that $R+$ $I Q_{c}=Q_{c}$. The second isomorphism theorem yields that $Q_{c} / I Q_{c} \simeq R /\left(I Q_{c}\right)^{c}$. The proof of (i) will be completed once it is shown that $I=\left(I Q_{c}\right)^{c} \cdot I Q_{c}=\left(I Q_{c}\right)^{c} Q_{c}$ so that by Lemma $2.16\left(I Q_{c}\right)^{c / I}$ is $c$-torsion. But $\left(I Q_{c}\right)^{c / l}$ is $b$-torsion since it is a submodule of $R / I$; therefore $\left(I Q_{c}\right)^{c / I}$ is both $c$-torsion and $b$-torsion and hence the zero module since the $c$-torsion cyclic modules are precisely the completely $b$-torsion free cyclic modules.

(ii) Suppose that $B$ is a two-sided ideal of $\mathcal{T}_{b}$ and that $Q_{c} B Q_{c}=Q_{c}$. Lemma 2.16 yields that $Q_{c} / B Q_{c}$ is c-torsion since $Q_{c}\left(B Q_{c}\right)=Q_{c}=Q_{c} Q_{c}$. In the proof (i) we have that $\left(B Q_{c}\right)^{c}=B$ and that $Q_{c} / B Q_{c} \simeq R / B$. The map giving the isomorphism is that of the second isomorphism theorem and in this case will be an $R-R$ bimodule map. In particular $Q_{c} / B Q_{c}$ is isomorphic to $R / B$ as a left $R$-module; $Q_{c} / B Q_{c}$ is thus b-torsion. $Q_{c} / B Q_{c}$ is both $b$-torsion and $c$-torsion and is therefore the zero module so that $Q_{c}=B Q_{c}$. But this contradicts the fact that $Q_{c} / B Q_{c} \simeq R / B$; whence $Q_{c} B Q_{c}=B Q_{c} \neq Q_{c}$ and similarly $Q_{c} B Q_{c}=Q_{c} B$. The fact that $Q_{c} / B Q_{c}$ and $R / B$ are isomorphic as rings follows as in Proposition 2.4.

We are now able to prove a proposition complementary to Corollary 2.9.

Proposition 2.18. Let $B$ and $C$ be simple right $R$-modules such that $B$ is $b$ torsion and $C$ is cbf (bence c-torsion). Then $\operatorname{Ext}_{R} 1$ (C, B) $=0$ 。

Proof. $\operatorname{Ext}^{1}(C, B)=0$ if and only if every short exact sequence

$$
0 \rightarrow B \rightarrow A \rightarrow C \rightarrow 0
$$

splits. Assume that $(*)$ is such a sequence that does not split. $B \simeq R / I$ where $I \in \mathfrak{T}_{b}$. By Proposition $2.17 B \simeq R / I \simeq Q_{c} / I Q_{c}$, a simple right $Q_{c}$-module; identify $B$ with $Q_{c} / I Q_{c} .(*)$ does not split so that $B$ is an essential submodule of $A$; hence $E(A)=E(B)$. Let $F$ be the $Q_{c}$-injective hull of $B . F$ is $Q_{c}$-divisible, thus $R$-divisible, and by a theorem of Levy [12] is then $R$-injective. Consequently $E(A)$ $C F . B$ is a $Q_{c}$-submodule of $F$ so that $F / B$ is a $Q_{c}$-module and hence is $c$-torsion free; this is a contradiction of the fact that $F / B$ is $c$-torsion. This implies that $B$ is not large in $A$ and that $(*)$ splits.

Corollary 2.9 and Proposition 2.18 are brought together in a stronger form in the following theorem.

Theorem 2.19. If $M$ is an Artinian finitely generated right (left) $R$-module, then $M=b(M) \oplus c(M)$. 
Proof. The proof will be by induction on the composition length of $M$. If the composition length of $M$ is one, then $M$ is simple and the result is trivially true. Suppose now that the result is valid for modules of composition length less than or equal to $n$, and also suppose that the composition length of $M$ is $n+1 . b(M) \cap$ $c(M)=0$ so it is sufficient to show that if $x \in M$, then $x=b+c$ where $b \in b(M)$ and $c \in c(M)$; this implies that it is sufficient to assume that $M$ is cyclic of composition length $n+1$. In this case $M \simeq R / I$ and there exists $I \subset L \subset R$ such that $R / L$ is simple and the composition length of $L / I$ is $n$. Throughout the rest of the proof we will refer to the following diagram:

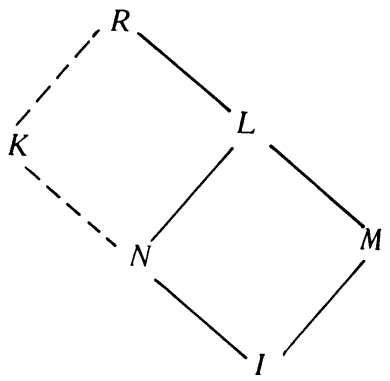

By the induction hypothesis there are right ideals of $R, N$ and $M$, such that $N \cap$ $M=I, N+M=L, N / I$ is $c$-torsion, and $M / I$ is $b$-torsion. $L / M \simeq N / I$ is $c$-torsion and $L / N \simeq M / I$ is $b$-torsion. There are two cases: either $R / L$ is $c$-torsion or $R / L$ $b$-torsion. We will consider the case in which $R / L$ is $c$-torsion; the proof in the other case is very similar. Again by the induction hypothesis (or if $N=I$ and the composition length of $R / I$ is 2, then by Proposition 2.18) $N=K \cap L^{\prime}$ where $K+$ $L^{\prime}=R, K / N$ is c-torsion, and $L^{\prime} / N$ is b-torsion. $K \cap M \supset N \cap M=I$. Also, $(K \cap M) / I$ is a submodule of $N / I$ and is thus $c$-torsion; at the same time however $(K \cap M) / I$ is a submodule of $M / I$ and thus is $b$-torsion. Therefore $(K \cap M) / I$ is the zero module and $K \cap M=I .(K+M) / I$ is isomorphic to $(K / I) \oplus(M / I)$ and therefore has composition length equal to that of $L / I$ plus one, that is, $n+1 . R / I$ also has composition length $n+1$ so that $K+M=R$. We have shown that $R / I=(K / I) \oplus$ $(M / I)$ where $K / I$ is $c$-torsion and $M / I$ is $b$-torsion which is the desired result.

As a corollary to Proposition 2.19 we have the next theorem which is a generalization of Theorem 3.9 of Eisenbud and Robson [4] since in a Dedekind prime ring the concepts of $c$-torsion and $b$-torsion reduce to those of completely faithful and bounded.

Theorem 2.20. Let $M$ be a right (left) torsion module over $R$. Then $M=b(M)$ $\oplus c(M)$.

Proof. It need only be shown that if $x \in M$, then $x=b+c$ where $b \in b(M)$ and $c \in c(M)$, for $b(M) \cap c(M)=0$. Take $x \in M ; x R$ is Artinian so that by Proposition $2.19 x=b+c$ where $b \in b(x R)$ and $c \in c(x R) . b(x R) \subset b(M)$ and $c(x R) \subset c(M)$; 
therefore $b \in b(M)$ and $c \in c(M)$ as desired.

The previous results are now used to deduce some further properties of the overring $Q_{c}$.

Proposition 2.21. If $I$ is an integral right $Q_{c}$-ideal, then $I^{c} \in \mathfrak{T}_{b}$ and $Q_{c} / I \simeq$ $R / I^{C}$.

Proof. Let $I$ be an integral right $Q_{c}$-ideal and consider $R / I^{c}$. By Proposition $2.19 R / I^{c}$ is the direct sum of its $b$-torsion submodule and its $c$-torsion submodule so that there are right ideals $J$ and $K$ of $R$ such that $J \cap K=I^{c}, J+K=R, R / J$ is $b$-torsion and $R / K$ is c-torsion. $R / K \simeq J / I^{c}$ by the second isomorphism theorem which means that $J / I^{c}$ is $c$-torsion. If $x \in J$, then there exists $L \in \mathfrak{T}_{c}$ such that $x L \subset I^{c} . x=x 1 \in x O_{l}(L)=x\left(L L^{*}\right)=x L L^{*} \subset I^{c} L^{*} \subset I^{c} Q_{c}$ and since $x$ was arbitrary in $J, J \subset I^{c} Q_{c}$; but $J \subset R$, therefore $J \subset I^{c} Q_{c} \cap R=I^{c}$. As a result $R / I^{c}=R / J$ is $b$-torsion. The fact that $Q_{c} / I \simeq R / I^{c}$ now follows from Proposition 2.17 .

A hereditary Noetherian prime ring is said to have enough invertible ideals if every nonzero two-sided ideal of $R$ contains an invertible two-sided ideal. The following theorem completes the structure of $Q_{c}$.

Theorem 2.22. $Q_{c}$ is a bounded bereditary Noetherian prime ring with enough invertible ideals. The one-sided (two-sided) $Q_{c}$-ideal lattices are isomorphic to the one-sided (two-sided) ideal lattices (lattice) of R-ideals in $\mathcal{J}_{b}$, and the corresponding factor modules (rings) are isomorphic.

Proof. Clearly $Q_{c}$ is hereditary and Noetherian, and the last statement is a statement of Propositions 2.17 and 2.21. It still must be shown that $Q_{c}$ is bounded with enough invertible ideals.

If $B$ is a two-sided invertible ideal of $R$, then $Q_{c} B=B Q_{c}$ is a two-sided ideal of $Q_{c}$. Consider $Q_{c} B^{-1} ; Q_{c} B^{-1}=Q_{c} R B^{-1}=R Q B^{-1}=B^{-1} B Q_{c} B^{-1}=B^{-1} Q_{c} B B^{-1}$ $=B^{-1} Q_{c} R=B^{-1} Q_{c}$; that is, $Q_{c} B^{-1}=B^{-1} Q_{c} \cdot\left(Q_{c} B^{-1}\right)\left(B Q_{c}\right)=Q_{c} R Q_{c}=Q_{c}=$ $\left(Q_{c} B\right)\left(B^{-1} Q_{c}\right)=\left(B Q_{c}\right)\left(Q_{c} B^{-1}\right)$; whence $B Q_{c}$ is an invertible two-sided ideal of $Q_{c}$.

Let $I$ be an integral right (left) $Q_{c}$-ideal. By Proposition $2.21 I^{c} \in \mathcal{T}_{b}\left({ }_{b} \mathcal{T}\right)$ and hence contains an invertible two-sided ideal $B$ of $R$. I then contains $B^{-1} Q_{c}$ an invertible two-sided $Q_{c}$-ideal. As a result $Q_{c}$ is bounded with enough invertible ideals.

Proposition 2.23. Let $B$ be a simple b-torsion right $R$-module. Then $E(B)$ is b-torsion.

Proof. The proof is much the same as those of Lemma 2.7 and Proposition 2.8.

The main results of this section are combined in the following theorem.

Theorem 2.24. Let $R$ be an hereditary Noetherian prime ring. Then $R$ is the intersection of a bounded bereditary Noetherian prime ring with enough invertible 
ideals and an bereditary Noetberian prime ring with no invertible ideals.

Proof. $R \subset\left(Q_{b} \cap Q_{c}\right)$ and the proof will be complete once it is shown that $Q_{b}$ $\cap Q_{c} \subset R$ and thus that equality holds. Take $x \in\left(Q_{b} \cap Q_{c}\right) \backslash R .[x+R]$ will then be an element of $Q / R$ which is both $c$-torsion and $b$-torsion. Hence $[x+R]=$ $[0+R]$ and $x \in R$.

3. Localizations at maximal invertible ideals. In this section it is shown that if $M$ is a maximal invertible ideal of an HNP ring $R$, then $R$ can be localized at $M$. That is, an overring of $R$ is constructed which is a bounded HNP with only finitely many maximal ideals. Also, the Jacobson radical of this overring is the expansion of $M$ and is invertible. Finally, it is shown that these localizations are classical in nature and that a globalization theorem holds.

Jategaonkar [9] has shown that localizations can be constructed at maximal invertible ideals in an HNP ring with enough invertible ideals, thus generalizing previous results of the author [10]. The results of this section generalize both those of Jategaonkar and the author.

Many of the proofs in this section are similar to those in $\$ 2$. When this occurs, it will be stated in order to keep repetitions at a minimum.

Let $M$ be a maximal invertible ideal of $R$. Let $\mathcal{T}_{m}=\{I: I$ is an integral right $R$-ideal and each composition factor of $R / I$ is annihilated by $M\}$. Clearly $I \in \mathcal{T}_{m}$ if and only if $I$ contains a power of $M$. If $A$ is a right $R$-module, define $m(A)$ by $m(A)=\left\{a \in A: a I=0\right.$ for some $\left.I \in \mathcal{T}_{m}\right\}$. The left analogs ${ }_{m} \mathfrak{T}$ and $m(B)$ for $B$ a right $R$-module are defined in a similar manner. A module $A$ is called completely $m$-torsion free if all submodules of all factor modules of $A$ are $m$-torsion free. Let $\mathcal{T}_{f}=\{I: I \text { is an integral right } R \text {-ideal and } R / I \text { is completely } m \text {-torsion free }\}_{.} I \in \mathfrak{T}_{f}$ if and only if $R / I$ has no $m$-torsion composition factors. If $A$ is a right $R$-module, $f(A)$ is defined by $f(A)=\left\{a \in A: a I=0\right.$ for some $\left.I \in \mathcal{T}_{f}\right\}$. Again $f \mathfrak{T}$ is defined in analogous fashion. Note that if $I \in \mathcal{T}_{f}$, then $I+M=R$. From this it follows that if $A$ is a right $R$-module, then $m(A) \cap f(A)=0$.

Proposition 3.1. $f$ and $m$ are idempotent kernel functors.

Proof. The proof is roughly the same as that of Lemma 2.10 and Proposition 2.12 .

The corresponding rings of quotients $Q_{m}$ and $Q_{f}$ are now formed. $Q_{m}$ is the union of the duals of all the right ideals in $\widetilde{T}_{m}$ while $Q_{f}$ is the union of the duals of all the right ideals in $\mathcal{T}_{f}$. The next proposition shows that these quotient rings are also the left-handed versions.

Proposition 3.2. $Q_{m}={ }_{m} Q$ and $Q_{f}={ }_{f} Q$.

Proof. $I \in \mathfrak{T}_{m}$ if and only if $I$ contains some power of $M$. Thus $Q_{m}$ is the union of all powers of $M^{-1}$; but ${ }_{m} Q$ is also the union of all powers of $M^{-1}$. Hence 
$Q_{m}={ }_{m} Q$. The proof of the fact that $Q_{f}={ }_{f} Q$ is much the same as that of Proposition 2.15 using a lemma similar to Lemma 2.14 .

We can now give some properties of $Q_{m}$.

Proposition 3.3. (i) If $I \in \mathcal{T}_{m}$, then $Q_{f} / I Q_{f} \simeq R / I$ and $I=\left(I Q_{f}\right)^{c}$.

(ii) If $B$ is a two-sided ideal in $\mathcal{T}_{m}$, then $Q_{f} B=Q_{f} B Q_{f}=B Q_{f}$, and $Q_{f} / B Q_{f}$ and $R / B$ are isomorphic as rings.

Proof. The proof is again essentially that of $\$ 2$.

Proposition 3.4. Let $S$ and $S^{\prime}$ be simple right $R$-modules such that $S$ is $m$ torsion and $S^{\prime}$ is f-torsion. Then $\operatorname{Ext}_{R}^{1}\left(S^{\prime}, S\right)=0$ and $\operatorname{Ext}_{R}^{1}\left(S, S^{\prime}\right)=0$.

Proof. The proof that $\operatorname{Ext}_{R}^{1}\left(S^{\prime}, S\right)=0$ is much the same as that of Proposition 2.18. $S^{\prime}$ is either $b$-torsion or $c$-torsion. If $S^{\prime}$ is $c$-torsion, then $\operatorname{Ext}_{R}^{1}\left(S, S^{\prime}\right)=0$ by Corollary 2.9. If $S^{\prime}$ is $b$-torsion, then $S^{\prime}$ is annihilated by some maximal invertible ideal $M^{\prime}$ and $M^{\prime} \neq M$. In this case $S$ if $f^{\prime}$-torsion and $S^{\prime}$ is $m^{\prime}$-torsion where $f^{\prime}$ and $m^{\prime}$ are the kernel functors. Hence $\operatorname{Ext}_{R}^{1}\left(S, S^{\prime}\right)=0$.

Proposition 3.5. Let $I$ be an integral right $Q_{f}$-ideal. Then $I^{c} \in \mathfrak{T}_{m}$ and $R / I^{c}$ $\simeq Q_{f} / I$.

Proof. The proof is essentially that of Proposition 2.21; it requires a lemma which is the analog of Proposition 2.20. We will not give it now since a generalization will be given later in the paper.

We are now ready to give the structure of the rings $Q_{m}$. The structure of $Q_{m}$ depends on the nature of the maximal invertible ideal M. By Theorem 2.6 of Eisenbud and Robson [3] either $M$ is a maximal ideal or $M$ is the intersection of a finite number of distinct idempotent maximal ideals $M_{1}, \cdots, M_{n}$ where $O_{r}\left(M_{1}\right)=O_{l}\left(M_{2}\right)$, $\cdots, O_{r}\left(M_{n}\right)=O_{l}\left(M_{1}\right)$. Such a set of maximal ideals is called a nontrivial cycle, or just a cycle.

Theorem 3.6. Let $M$ be a maximal invertible ideal of an $H N P$ ring $R$, and let $m$ and $f$ be the associated kernel functors. Then $Q_{f}$ is a bounded HNP with enough invertible ideals whose Jacobson radical is $M Q_{f}$. Furthermore:

(i) If $M$ is a maximal ideal, then $Q_{f}$ is a Dedekind prime (in fact a PIR) with a unique maximal ideal $M Q_{f}$. Every ideal of $Q_{f}$ is a power of $M Q$.

(ii) If $M$ is an intersection of a cycle, say $M=M_{1} \cap \cdots \cap M_{n}$ where $M_{1}, \cdots$, $M_{n}$ is a cycle, then $M Q_{f}=M_{1} Q_{f} \cap \ldots \cap M_{n} Q_{f}$ where $M_{1} Q_{f}, \cdots, M_{n} Q_{f}$ is a cycle. In fact the $M_{i} Q_{f}$ 's are the only maximal ideals of $Q_{f}$ and all are idempotent. Also, every invertible ideal of $Q_{f}$ is a power of $M Q_{f}$.

Proof. $Q_{f}$ is bounded since by Proposition 3.5 if $I$ is an integral right $Q_{f}$ ideal, then $I^{c} \in \mathcal{J}_{m}$ so that $I^{c}$ contains some power, $M^{k}$, of $M$. I then contains the two-sided ideal of $M^{k} Q_{f}$. 
(i) Let $I$ be a two-sided ideal of $Q_{f} ; I^{c}$ is then a two-sided ideal in $\mathcal{J}_{m}$ and hence contains some power of $M$; suppose that $k$ is the least such power. We show by induction on $k$ that if $J$ is any $R$-ideal with $k$ the least positive integer such that $M^{k} C$ $J$, then $M^{k}=J$. If $k=1$, then $M \subset J \subset R$ so that $M=J$ since $M$ is a maximal ideal. Inductively suppose that the result holds for all powers less than $k$ and that $k$ is the least positive integer such that $M^{k} \subset J$. $J$ must be contained in $M$ so that $M^{-1} M^{k} \subset M^{-1} J \subset M^{-1} M$ and $M^{k-1} \subset M^{-1} J \subset R$. Then the least power of $M$ contained in $J$ is no bigger than $k-1$ and the induction hypothesis implies that $J$ is a power of $M$. It follows that $I^{c}=M^{k}$ for some $k$. Every ideal is the power of its contraction, and $M Q_{f}=Q_{f} M$; therefore $I=M^{k} Q_{f}=\left(M Q_{f}\right)^{k}$ and every ideal is a power of $M Q_{f} . M Q_{f}$ is the Jacobson radical of $Q_{f}$ since it is the unique maximal ideal of $Q_{f}$ and since $Q_{f}$ is bounded. As in the case of $Q_{c}$, expansions of invertible ideals are invertible and $\left(M^{k} Q_{f}\right)^{-1}=Q M^{-k} Q_{f}$. This means that $Q_{f}$ is a Dedekind prime ring. Let $I$ be an integral right $Q_{f}$-ideal; then there is a power $k$ of $M Q_{f}$ which is properly contained in $I$, in fact small in $I$ since $M Q_{f}=\operatorname{rad}\left(Q_{f}\right)$. $Q_{f} /(M Q)^{k}$ is an Artinian PIR so that $I /\left(M Q_{f}\right)^{k}$ is principal. $\left(M Q_{f}\right)^{k}$ is small in $I$ which implies that $I$ is principal and that $Q_{f}$ is a PIR.

(ii) Every ideal is the expansion of its contraction and every ideal in $\mathcal{T}_{m}$ has a proper expansion, therefore the maximal (two-sided) ideals of $Q_{f}$ are precisely $M_{1} Q_{f}, \cdots, M_{n} Q_{f}$. Since $Q_{f} / M Q_{f} \simeq R / M, \cap M_{i} Q_{f}=M Q_{f} ; Q_{f}$ is bounded so that $M Q_{f}$ is the Jacobson radical of $Q_{f}$.

First we note that an ideal $I$ of $Q_{f}$ is idempotent if and only if its contraction $I^{c}$ is idempotent in $R$, for $I=I^{2}=\left(I^{c} Q_{f}\right)\left(I^{c} Q_{f}\right)=\left(I^{c}\right)^{2} Q_{f}$ and $\left(\left(\left(I^{c}\right)^{2}\right) Q_{f}\right)^{c}=\left(I^{c}\right)^{2}=$ $I^{c}$. This also means that if $J$ is an idempotent ideal in $\mathcal{J}_{m}$, then $J Q_{f}$ is idempotent. Clearly then an ideal of $Q_{f}$ is eventually idempotent if and only if its contraction is eventually idempotent. Note also that all powers of $M Q_{f}$ are invertible since $M^{-k} Q_{f}=Q_{f} M^{-k}$ and $\left(Q_{f} M^{-k}\right)\left(M^{k} Q_{f}\right)=Q_{f}=\left(Q_{f} M^{k}\right)\left(M^{-k} Q_{f}\right)$. These are in fact the only invertible ideals of $Q_{f}$; for assume that $I$ is an invertible ideal of $Q_{f}$, then $I^{c}$ contains some power of $M$, say $M^{k}$. If $k=1$, then either $I^{c}=M$ (in which case $\left.I=M Q_{f}\right)$ or else $I^{c}$ properly contains $M . M$ is a maximal invertible ideal which implies that $I^{c}$ is eventually idempotent and hence by the above that so is $I$. But this contradicts the fact that $I$ is invertible; therefore, $I^{c}=M$. Inductively assume that if $J$ is any invertible ideal of $Q_{f}$ such that the least power of $M$ contained in $J^{c}$ is less than the $k$ th, then $J$ is a power of $M Q_{f}$. Suppose that $I$ is an invertible ideal of $Q_{f}$ and that $M^{k}$ is the least power of $M$ contained in $I^{c}$ with $k$ greater than one. $\left(M Q_{f}\right)^{k}$ is then an invertible ideal contained in $I$. I being an invertible ideal is contained in a maximal invertible ideal of $Q_{f}$, say $L . L$ is a finite intersection of idempotent maximal ideals and hence must contain $M Q_{f}$ the intersection of all the maximal ideals. In this case $L^{c} \supset M$ and by the induction hypothesis $L^{c}$ $=M$ and $L=M Q_{f}$. Hence $\left(M Q_{f}\right)^{k} \subset I \subset M Q_{f}$ and $\left(M Q_{f}\right)^{k-1} \subset\left(M Q_{f}\right)^{-I} I \subset Q_{f}$. 
$\left(M Q_{f}\right)^{-1} I$ is again invertible and the least power of $M$ contained in $\left(\left(M Q_{f}\right)^{-1} I\right)^{C}$ is less than $k$ so that $\left(M Q_{f}\right)^{-1} I$ is a power of $M Q_{f}$ and hence so is $I$.

Let $S=\{m: m$ is the kernel functor associated to a maximal invertible ideal M). The following theorem gives a decomposition theory for torsion modules over a hereditary Noetherian prime ring.

Theorem 3.7. Let $A$ be a torsion right $R$-module. Then $A=c(A) \oplus\left(\bigoplus_{S} m(A)\right)$.

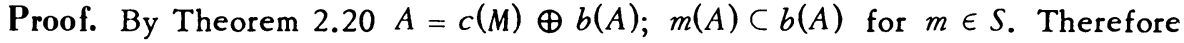
it just remains to be shown that $b(A)=\bigoplus_{S} m(A)$. Also $m(A) \cap\left(\bigoplus_{m^{\prime} \neq m} m^{\prime}(A)\right)=0$ since $\bigoplus_{m^{\prime} \neq m} m^{\prime}(A) \subset f(A)$ where $f$ is associated with $M$. Hence it is enough to show that every element of $b(A)$ is a sum of elements of the desired type, or assume that $b(A)$ is cyclic and hence of finite composition length. From these, one can proceed by induction on the composition length. If the length is one, the result is clear. If not, then a proof similar to that of Proposition 2.19 shows that if $m_{1}$ is such that $b(A)$ has at least one $m_{1}$-torsion composition factor then $b(A)=m_{1}(b(A))$ $\oplus f_{1}(b(A))$ and $f_{1}(b(A))$ has composition length strictly less than that of $b(A)$ and the result follows from the induction hypothesis.

Let $M$ again be a maximal invertible ideal in the HNP ring $R$. Define the cancellation set of $M, C(M)$, by $C(M)=\{b \in R: b x \in M$ implies $x \in M\}$. Hence $b \in$ $C(M)$ if and only if $[b+M]$ is right regular in $R / M . R / M$ is a semisimple Artinian ring so that in $R / M$ being right regular is equivalent to being a unit which is equivalent to being left regular. As a result we have that $C(M)=\{b \in R: x b \in M$ implies $x \in M\}$. Also, $C(M)=\{b \in R: b R+M=R\}=\{b \in R: R b+M=R\}$.

Let $b \in C(M)$; then $b R+M=R$ which implies that $b M+M^{2}=M$. Hence $b R+$ $\left(b M+M^{2}\right)=R$ and $b R+M^{2}=R$. Induction yields that $b R+M^{n}=R$ for all $n$; that is, $\left[b+M^{n}\right]$ is a unit in $R / M^{n}$ for all $n$. Suppose that $b x=0$; this means then that $x \in M^{n}$ for all values of $n$. Since $M$ is invertible, the intersections of its powers is zero; and hence that $x$ is zero. As a result, all the elements of $C(M)$ are regular.

Lemma 3.8. If $b \in C(M)$, then $R / b R$ is f-torsion; that is, $R / b R$ has no compos. tion factors which are annibilated by $M$.

Proof. Consider $R / b R . R / b R=f(R / b R) \oplus m(R / b R)$ by 3.7. Equivalently, there are right ideals $I$ and $J$ of $R$ such that $I \cap J=b R, I+J=R, R / I$ is $f$-torsion and $R / J$ is $m$-torsion. If $R / b R$ is not f-torsion, then $J \neq R$; hence $b R+M \subseteq$ $J+M \neq R$ which contradicts the fact that $b \in C(M)$. Hence $R / b R$ is f-torsion.

Lemma 3.9. Let $I \in \mathcal{T}_{f}$. Then $I$ contains an element of $C(M)$.

Proof. $R / I$ is $f$-torsion so that $R / I$ has no composition factors annihilated by $M$. Therefore $I+M=R$, and hence $1=x+m$ for $x \in I$ and $m \in M . x R+M=R$ so that $x \in C(M)$. 
Theorem 3.10. $Q$, is a classical localization of $R$. That is, every element of $Q$, can be written in the form $x b^{-1}\left(d^{-1} y\right)$ where $x \in R$ and $b \in C(M)(y \in R$ and $d \in C(M)$ ); every element of $C(M)$ bas its inverse in $Q_{f} . R$ satisfies the Ore conditions with respect to $C(M)$.

Proof. If $b \in C(M)$, then $b^{-1} \in Q$, by Lemma 3.8. Conversely let $q \in Q_{f}$, then $q \in I^{*}$ for $I \in \mathcal{T}_{f}$. Then there is $b \in C(M)$ such that $b \in I$. $b R \subset I$ so that $R b^{-1} \supset I^{*}$. As a result $q=x b^{-1}$ for $x \in R$. The other representation follows in similar fashion. The Ore conditions are forced by the fact that $Q_{f}$ is a ring.

Lemma 3.11. Let $\sigma$ be an idempotent kernel functor in $\operatorname{Mod}\left(R_{R}\right)$ where $R$ is an $H N P$ ring. Then $\sigma(B)=\operatorname{ker}\left(B \rightarrow B \otimes_{R} Q_{\sigma}\right)$ where $Q_{\sigma}$ is the ring of quotients with respect to $\sigma$.

Proof. $Q_{\sigma}$ is an overring of $R$ and is the union of the duals of the elements of $\mathcal{J}_{\sigma}$ by the results of section one. By ${ }^{[10]}{ }_{R} Q_{\sigma}$ is flat so that we can just show that a right ideal $I$ is in $\mathcal{T}_{\sigma}$ if and only if $(R / I) \otimes Q_{\sigma}=0(x \in \sigma(B)$ if and only if $x R \simeq R / I$ for some $\left.I \in \mathfrak{T}_{\sigma}\right)$. The key to the fact is the following commutative diagram where the horizontal maps are inclusion maps and the vertical maps are defined by $\Sigma x_{i} \otimes y_{i} \rightarrow \sum x_{i} y_{i}$

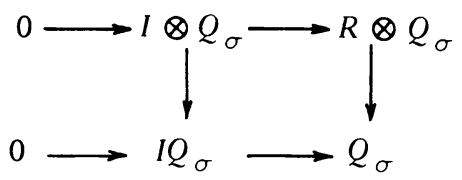

The vertical maps are in fact isomorphisms since $Q \otimes_{R} Q \simeq Q$ under the same map. $(R / I) \otimes Q_{\sigma}=0$ if and only if the map of the top row is onto. But this happens exactly when the bottom map is onto which is when $I Q_{\sigma}=Q_{\sigma}$. However, this holds precisely when $I \in \mathcal{J}_{\sigma}$.

We now can prove the globalization theorem.

Theorem 3.12. Let $B$ be a right $R$-module such that $B \otimes_{R} Q_{b}=0$ and $B \otimes_{R} Q_{f}=0$ for each maximal invertible ideal $M$. Then $B$ is the zero module.

Proof. All of the overrings involved are flat as left $R$-modules, therefore we may assume that $B$ is a cyclic torsion module. Since $B \otimes Q_{b}=0, B$ is $b$-torsion by Lemma 3.12. Since tensor products commute with direct sums we may in view of Theorem 3.7, assume that $B$ is $m$-torsion where $m$ is associated with the maximal ideal $M$. Let $f$ be associated with $M$, then $B \otimes Q_{f}=0$ so that by Lemma 3.11 $B$ is $f$-torsion. Hence $B$ is both $f$ and $m$-torsion and is thus the zero module.

4. The one and one-half generator property. In this section it is shown as an application that if $R$ (an HNP) has the one and one-half generator property for integral right $R$-ideals, then $R$ is a Dedekind prime ring. The main tool used will be 
the localization technique. Eisenbud and Robson [4] have shown that the one and one-half generator property holds for Dedekind prime rings.

$R$ is said to have the one and one-half generator property for integral right $R$ ideals if given an integral right $R$-ideal $I$ and $b$ a regular element of $I$, then $I / b R$ is principal. Another way of stating this is that $I$ is generated by two elements, one of which may be chosen almost at random. It might also be noted that for HNP's this just means that submodules of cyclic torsion modules are cyclic.

Eisenbud and Robson [3] have shown that an HNP ring will be Dedekind if and only if it has no idempotent maximal ideals. For the rest of the section $R$ will be an HNP ring with the one and one-half generator property and $M$ will be idempotent maximal ideal of $R$. The proof will be complete once it is shown that the fact that $M$ is idempotent leads to a contradiction.

Let $m$ be the kernel functor determined by $M$ and let $f$ be the associated kernel functor determined by the set of integral right $R$-ideals $I$ such that $R / I$ is completely $m$-torsion free.

Lemma 4.1. I $\in \mathcal{T}$, if and only if $I+M=R$.

Proof. If $\in \mathcal{T}_{f}$, then $R / I$ has no $m$-torsion composition factors and hence $I+$ $M=R$.

Conversely, let $I$ be such that $I+M=R$. If $I+M=R$, then $L+M=R$ for any $L \supset I$; therefore, we can assume that $I \subset J \subset R$ such that $J \in \mathcal{J}_{f}$ and $J / I$ is a simple $m$-torsion module. Consider the following diagram:

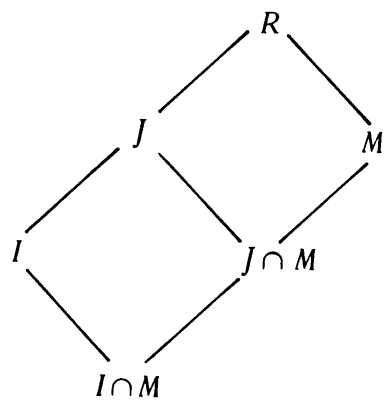

$R / M \simeq I /(I \cap M)$ and $J / I$ is cyclic $m$-torsion. Hence if the composition length of $R / M$ is $k$, then the composition length of $J /(I \cap M)$ is $k+1$. Each composition factor of $J /(I \cap M)$ is $m$-torsion so that $(J / I \cap M) M^{k+1}=0=(J / I \cap M) M$ since $M$ is idempotent. This says that $J /(I \cap M)$ is an $R / M$-module. By the one and onehalf generator property $J /(I \cap M)$ is cyclic as a right $R$-module; therefore, it is cyclic as an $R / M$-module. This, however, is a contradiction since cyclic modules over $R / M$ can have composition length at most $k$. Thus it must be that $J=I$ and $I \in \mathcal{T}_{f}$. 
Lemma 4.2. Let $I \in \mathcal{T}_{f}$, then there is a regular $a \in I$ such that $a R \in \mathcal{J}_{f}$.

Proof. $I \in \mathcal{T}_{f}$ so that $I+M=R . R$ has the one and one-half generator property so that there is an element $a$ of $I$ such that $a R+I \cap M=I$. Hence $a R+M=R$ and $a R \in \mathfrak{T}_{f}$.

Again we could form the analogous left kernel functors $m$ and $f$ with associated filters of left ideals ${ }_{m} \mathfrak{J}$ and, $\mathcal{T}$. The next proposition relates the left and right theories; namely, their rings of quotients coincide.

Proposition 4.3. $Q_{f}=, Q$ and every element of $Q_{f}$ can be expressed in the form $a^{-1} r$ or $s b^{-1}$ where $a, b, r$, and $s$ are elements of $R$ and $a R+M=R=R b+M$.

Proof. $Q_{f}$ is the union of all $I^{*}$ where $I \in \mathfrak{T}_{f}$; but by Lemma $4.2 a R \subset I$ where $a R+M=R$. Thus $I^{*} \subset R a^{-1}$ and $Q$ is the union of all such $R a^{-1}$. Similarly $f_{f}$ is the union of all $b^{-1} R$ where $R b+M=R$. But $R b+M=R$ just says that $[b+M]$ has a left inverse in the simple Artinian ring $R / M$; hence $[b+M]$ has a right inverse and $b R+M=R$. As a result $b^{-1} \in Q_{f}$ and hence $b^{-1} R \subset Q_{f}$. Thus ${ }_{f} Q \subset Q_{f}$; similarly $Q_{f} \subset, Q$ and $Q_{f}={ }_{f} Q$.

In the next proposition we give a few properties of $Q_{f}$. as rings.

Proposition 4.4. (i) $M Q_{f}=Q_{f} M Q_{f}=Q_{f} M$ and $Q_{f} / M Q_{f}$ and $R / M$ are isomorphic

(ii) $M Q_{f}$ is the unique maximal two-sided ideal of $Q_{f}$.

(iii) $M Q_{f}$ is the Jacobson radical of $Q_{f}$.

Proof. Suppose that $Q_{f} M Q_{f}=Q_{f}$. Then $\Sigma q_{i} m_{i} p_{i}=1$ where the $m_{i}$ 's are in $M$, and the $q_{i}$ 's and $p_{i}$ 's are in $Q_{f}$. Then there are elements $a$ and $b$ in $R$ such that $R a+M=R$ and $b R=M=R$ for which $q_{i}=a^{-1} r_{i}$ for all $i$ and $p_{i}=s_{i} b^{-1}$ for all $i$ where the $r_{i}$ 's and $s_{i}$ 's are in $R$. Then $1=\sum a^{-1} r_{i} m_{i} s_{i} b^{-1}$ and $a b=$ $\Sigma r_{i} m_{i} s_{i}$ is an element of $M$. This contradicts, however, the fact that $[a+M]$ and $[b+M]$ are units in $R / M$. Therefore $Q_{f} M Q_{f} \neq Q_{f} \cdot\left(Q_{f} M Q_{f}\right)^{c}=M$ since $M$ is maximal and $\left(Q_{f} M Q_{f}\right)^{c}$ is a proper two-sided ideal of $Q_{f}$ that contains $M$. Every ideal is the expansion of its contraction; therefore, $M Q_{f}=Q_{f} M Q_{f}=Q_{f} M$. The last statement easily follows.

(ii) Let $I$ be a maximal two-sided ideal of $Q_{f}$. Then $I^{c}$ is a two-sided ideal of $R$ and $I^{C}+M=M$ and $I=I^{C} Q_{f} \subset M Q_{f}$. Hence $I=M Q_{f}$ since $I$ is a maximal twosided ideal of $Q_{f}$.

(iii) Let $I$ be an integral right $Q_{f}$-ideal and let $J=I^{c}+M . J \neq M$ since if this were the case, $I=I^{c} Q_{f}$ would have to be all of $Q_{f}$. Claim. $J Q_{f} \neq Q_{f}$. Suppose that $J Q_{f}=Q_{f}$. Then $\sum x_{i} q_{i}=1$ where the $x_{i}$ 's are in $J$ and the $q_{i}$ 's are in $Q_{f}$. Then there is a regular element $b$ of $R$ such that $q_{i}=r_{i} b^{-1}$ for $r_{i}$ 's in $R$. Hence $1=$ $\sum x_{i} q_{i}=\sum x_{i} r_{i} b^{-1}=\left(\sum x_{i} r_{i}\right) b^{-1}$ and $b=\sum x_{i} r_{i}$ an element of $J$. If this were the case, however, then $b R+M \subset J+M \subset J$ contradicting the fact that $b R+M=R$; 
hence $J Q_{f} \neq Q_{f} . J Q_{f} \supset I^{c} Q_{f}=I$ a maximal right ideal of $R$; hence $J Q_{f}=I . J \supset M$ so that $I=J Q_{f} \supset M Q_{f}$. $I$ was an arbitrary maximal right ideal; therefore $M Q_{f}$ is the Jacobson radical of $Q_{f}$ by (ii).

We are now ready to prove the main result of this section.

Theorem 4.5. A bereditary Noetherian prime ring with the one and one-balf generator property is a Dedekind prime ring.

Proof. It is enough to show that if $R$ is such a ring, then $R$ has no idempotent maximal ideals. Suppose that $M$ is an idempotent maximal ideal of $R$. Form the ring $Q_{f}$ constructed earlier in the section. $Q_{f}$ is hereditary and in particular Noetherian. $M Q_{f}$ was also shown to be the Jacobson radical of $Q_{f}$; hence by Nakyama's lemma $M Q_{f}$ is not idempotent. But if $M$ is idempotent, then $\left(M Q_{f}\right)^{2}=\left(M Q_{f}\right)\left(M Q_{f}\right)=$ $M^{2} Q_{f}=M Q_{f}$, a contradiction. Therefore $R$ has no idempotent maximal ideals and $R$ is a Dedekind prime ring.

5. Remarks. In this section the results of the previous sections are compared with known results and related examples are given.

A ring is called pre-QF if every proper homomorphic image of it is quasi-Frobenius (QF). For commutative rings Levy has shown that a non-Artinian commutative pre-QF ring is a Dedekind domain. While it is true that every Dedekind prime ring is a pre-QF ring, Robson [14] has recently given an example of a hereditary Noetherian prime ring with precisely one two-sided ideal; hence even in the class of HNP rings a ring can be pre-QF without being Dedekind. The one and one-half generator property of $\$ 4$ is a strengthening of the hypothesis of being pre-QF. Robson's example shows that the hypothesis of $\$ 4$ cannot be weakened to pre-QF.

A module is called bounded if every element of it is annihilated by a nonzero two-sided ideal of the ring; a module is called completely faithful if every submodule of every factor module is faithful (see [4]). If $R$ is an HNP with enough invertible ideals, then these concepts are equivalent to being $b$-torsion and c-torsion respectively. Hence if $R$ is an HNP with enough invertible ideals, then Theorem 2.20 coincides with Theorem 3.9 of [4] which says that every torsion module is the direct sum of a bounded module and a completely faithful module; our proof, however, is different. The above example shows that this theorem cannot be proven for an arbitrary HNP ring; the argument is given below.

Let $R$ be an HNP ring with a unique two-sided ideal $M$ such that every torsion module over $R$ splits into a direct sum of a completely faithful module and a bounded module. Note that the class of completely faithful modules forms a torsion class and that its associated filter $\mathcal{T}_{c f}$, of right ideals is given by $\mathcal{T}_{c f}=\{I \subset R: R / I$ is completely faithful $\}=\{I \subset R: R / I$ has no bounded composition factors $\}$. It easily follows from the assumed splitting property that $\mathcal{J}_{c f}=\{I \subset R: I+M=R\}$. Form the associated ring of quotients $Q_{c f}$. Then again the assumed splitting property shows 
that if $I$ is a maximal right ideal of $Q_{c f}$, then $I^{c}=I \cap R$ contains $M$. Hence the intersection of all maximal right ideals of $Q_{c f}$ has nonzero Jacobson radical J.R has only one two-sided ideal, $M$, and $J^{c}$ is a two-sided ideal; therefore $J=M Q_{c f}=$ $Q_{c f} M$. Then $J^{2}=\left(M Q_{c f}\right)\left(Q_{c f} M\right)=M Q_{c f} M=M^{2} Q_{c f}=M Q_{c f}=J$; but $J$ is the Jacobson radical of a Noetherian ring and therefore cannot be idempotent by Nakyama's lemma. Hence we have a contradiction, and such a ring cannot enjoy such a splitting property.

\section{REFERENCES}

1. G. M. Bergman, Commuting elements in free algebras and related topics in ring theory, Thesis, Harvard University, Cambridge, Mass., 1967.

2. S. Dickson, A torsion theory for abelian categories, Trans. Amer. Math. Soc. 121 (1966), 223-235. MR 33 \#162.

3. D. Eisenbud and J. C. Robson, Hereditary Noetherian prime rings, J. Algebra 16 (1970), 86-104.

4. - Modules over Dedekind prime rings, J. Algebra 16 (1970), 67-85.

5. L. Fuchs, l'orsion preradicals und ascending Loewy series of modules, J. Reine Angew. Math. 239 (1969), 169-179.

6. P. Gabriel, Des catégories abéliennes, Bull. Soc. Math. France 90 (1962), 323-448. MR 38 \#1144. \#6914.

7. O. Goldman, Rings and modules of quotients, J. Algebra 13 (1969), 10-47. MR 39

8. N. Jacobson, The theory of rings, Math. Surveys, vol. 1, Amer. Math. Soc., Providence, R. I., 1943. MR 5, 31.

9. A. V. Jategaonkar, Unpublished letter.

10. J. Kuzmanovich, Localizations of Dedekind prime rings, J. Algebra 21 (1972), $378-393$.

11. J. Lambek, Torsion theories, additive semantics, and rings of quotients, Lecture Notes in Math., no. 177, Springer-Verlag, Heidelberg, 1971.

12. L. Levy, Torsion-free and divisible modules over non-integral domains, Canad. J. Math. 15 (1963), 132-151. MR 26 \#155.

13. J. M. Maranda, Injective structures, Trans. Amer. Math. Soc. 110 (1964), 98-135. MR 29 \#1236.

14. J. C. Robson, Idealizers and hereditary Noetherian prime rings, J. Algebra 22 (1972), 45-81. \#177.

15. …, Non-commutative Dedekind rings, J. Algebra 9 (168), 249-265. MR 38

16. C. Walker and E. Walker, Quotient categories and rings of quotients, Rocky Mountain J. Math 2 (1972), 513-555.

17. D. B. Webber, Ideals and modules of simple Noetherian hereditary rings, J. Algebra 16 (1970), 239-242. MR $42 \# 305$.

DEPARTMENT OF MATHEMATICS, UNIVERSITY OF NORTHERN COLORADO, GREELEY, COLORADO 80631

Current address: Department of Mathematics, Wake Forest University, Winston-Salem, North Carolina 27109 